\title{
BER performance study for optical OFDM of optical camera communication
}

\author{
Noor J. Jihad ${ }^{1}$, Sinan M. Abdul Satar ${ }^{2}$ \\ ${ }^{1}$ University of Technology, Communication Engineering Department, Baghdad, Iraq \\ ${ }^{2}$ University of Technology, Electrical and Electronic Engineering Department, Baghdad, Iraq
}

\begin{tabular}{|c|c|}
\hline Article Info & ABSTRACT \\
\hline Article history: & In this article, different forms of optical orthogonal frequency division \\
\hline Received Dec 29, 2020 & $\begin{array}{l}\text { multiplexing (OFDM) were observed which were suitable for optical camera } \\
\text { communication (OCC) systems. This research aims to establish the bit error }\end{array}$ \\
\hline Revised Apr 9, 2021 & rate (BER) versus signal-to-noise ratio (SNR) of the OCC system. This \\
\hline Accepted Apr 20, 2021 & $\begin{array}{l}\text { research will focus on OCC systems and the design that produces the noise of } \\
\text { the clipping but will gain SNR as a whole if an optimum clipping factor is }\end{array}$ \\
\hline Keywords: & $\begin{array}{l}\text { chosen. The BER versus SNR analysis was investigated for the different } \\
\text { clipping factors } 0.7,1.4 \text {, and } 2.6 \text {. The BER performance of the asymmetrically }\end{array}$ \\
\hline ACO-OFDM & clipped optical OFDM (ACO-OFDM) was also compared with the direct \\
\hline BER & $\begin{array}{l}\text { current optical OFDM (DCO-OFDM) to show the suitable effectiveness of the } \\
\text { proposed approach. ACO-OFDM was considered to be better due to lower bit }\end{array}$ \\
\hline Clipping factor & loading, but DCO-OFDM was efficient for higher SNR values. This was \\
\hline DCO-OFDM & because the DC bias used was inefficient in terms of optical capacity, while \\
\hline OCC & ACO-OFDM used only half of the subcarriers to transmit the information. \\
\hline SNR & $\begin{array}{l}\text { Moreover, ACO-OFDM two-dimensional half-subcarriers of mapping rule } \\
\text { would introduce the clipping noise to its unused } 2 \mathrm{D} \text { subcarriers, although } \\
\text { further data can be provided by the } 2 \mathrm{D} \text { DCO-OFDM mapping rule. }\end{array}$ \\
\hline
\end{tabular}

This is an open access article under the CC BY-SA license.

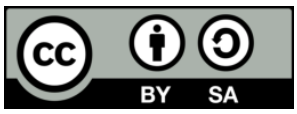

\section{Corresponding Author:}

Noor J. Jihad

Communication Engineering Department

University of Technology

Baghdad, Iraq

Email: noor.j.jihad@uotechnology.edu.iq

\section{INTRODUCTION}

In recent years, exponential demand for wireless communication systems for several businesses had increased, and spending much of their hunting budgets for new technologies to make their consumption easier for potential market control or consumer satisfaction demand. Wireless technology "out-of-the-box" is expected to have tremendous potential for the market. This technology was based on the free spectrum of radio interference, which means optical wireless communications (OWC) [1]. The OWC business shared the massive traditional financial distributors in the recent IEEE standards. IEEE release 802.15.7-2011 was maybe not successful until that time since there were no profitable products that would be pro-belonged to these collections. On the other hand, LiFi which allowed improved efficiency and demands more complex output waveform and protocols should apply to low-market specialty quantities [2]. Such technologies as visible light communication (VLC) and light fidelity LiFi were worth learning. However, testing at the optical camera communication (OCC) had been supposed more appealing for this research, not only because of the enormous volume of its applications but also provided a wide range of useful system possibilities of management, software, and the prototype of those systems could be established without any serious concern about the 
hardware design [3]. A classification of OCC technologies was most influenced by camera quality models. The output barrier of the OCC system was caused by its communication that supports the main imaging aimed at the camera [4].

The screen OCC structures are mainly presented in this report. It provides a newly intended screen code enabling 2-dimensional orthogonal frequency division multiplexing (2D-OFDM). The applications for two-dimensional code, including statistics QR code model, had disappeared from the market since the need for higher data delivery could not be met. All of these problems might be resolved by the dynamic QR model. However, from this analysis and QR code implementation including an Implementation of tri-color sequence transmission. The present code does not meet with the QR code, because of the enormous overhead sum per code and extended delay time, we considered the necessity for continuous protocol communication. A simultaneous QR device that offers no more than five codes per second on a personal computer PC [5] can also be configured. Good results must essentially be obtained from a modern 2D code architecture, close to QR in complex circumstances, vital characteristics, effective and fast detection, support for monitoring, rotation, and variety compatibility of screen and camera technology. The OFDM concept had introduced to an advanced maturity standard and work in the 1990s [6].

It is known that the multi-carrier modulation (MCM) of OFDM has multiple advantages compared to the conventional modulation schemes for single-carrier basebands, for example, on-off keying (OOK) [7]. This means, the stream of data an individual carrier that was orthogonal to other carriers transfer the higher data rates stream (space-time domain) into a lower bit rate [8]. Potentially this attractive feature is needed to adjust a channel equalizer to the proper configuration of the proposed system. The frame rate is usually 60 frames per second for smartphones, a 30 bit per second data rate while using a single light-emitting diode (LED), which is suitable for applications with a low data rate, such as placement and car-to-car communication [9]. The aim of this work basically to verify that the signal was being generated correctly and what factors would affect system performance if a back-to-back transmitter and receiver (bidirectional communication system) was connected. In the particular example of usage for 2D asymmetrically clipped optical OFDM (2D ACO-OFDM) and 2D direct current optical OFDM (2D DCO OFDM), this article will concentrate on the OCC system and its implementation. The analysis will refer to two parts, the research part of the optical OFDM system and OCC implementation, and the other part, which will address bit error rate (BER) performance for 2D ACO-OFDM and 2D DCO OFDM.

\section{MAJOR CONTRIBUTIONS}

The Optical OFDM OCC system was not a new invention, but there were some significant contributions to the present scheme which can be analyzed as:

- The corresponding size of the 2D-OFDM transmitter was based on several test verifications.

- A special and single code transmitter to promote the essential features which were hardly considered in the associated studies.

- FEC forward error correction technique: a rapid correction of the spatial code distortion. This technique supporting maximum accuracy in optical communication.

- A MIMO multi input multi output channel involving the various sub-channels for multi- subcarriers for the sequential distribution of data through the domain of space-frequency.

- Guidelines of receiver decoding and processing.

- Two-dimensional ACO OFDM and DCO OFDM clipping processes at different clipping factors to test the BER performance of the OCC system.

- One of the second generations of forward error correction (FEC) that was tested and applied in this framework was a successful FEC, therefore normally specific to the system and associated with the IEEE guidelines association's optical wireless communications (OWC) standards.

\section{OPTICAL OFDM SYSTEM}

Optical wireless communication (OWC) was a successful standard for developing the speeds of data transfer in the transmission medium. Infrared and visible light communication was able to offer several hundred Mbps [10]. In addition to providing a compatible approach that does not conflict with RF technologies, OWC gains from license-free operating over a considerably broader range of products [11]. Intensity modulation and direct detection (IM/DD) guarantee information transfer in OWC [12].

A real, non-negative waveform stimulates the intensity of the transmitter light-emitting diode (LED) and is detected by something like a photodiode detector (PD) at the receiver [13]. As compared to traditional optical OFDM systems, all-optical OFDM systems that use high order modulation formats seem to be more 
desirable because they can transfer information at a higher bit rates. [14]. Two potential systems activities of O-OFDM could be found in the literature: DCO-OFDM and ACO-OFDM when Hermitian symmetry is implemented on the OFDM subcarriers, it obtains a truly evaluated signal [15]. The DCO-OFDM provides a non-negative signal by adding a direct current (DC) systematic error [16].

\subsection{D DCO-OFDM}

The basic functional diagram of the DCO-OFDM system is specified as follows. QAM mapping is utilized to map bits into a complex set of numbers in one-dimensional order. A bi-dimensional matrix of complex numbers from a set of complex numbers can be assembled by Hermitian mapping in such a way that 2D-IDFT could be a real number. Therefore, DCO-OFDM assigns details to all 2D Hermitian matrix subcarriers, unlike ACO-OFDM [17]. The main purpose of adding the cyclic prefix CP is to avoid the OFDM symbols from overlapping with the other symbols. This DCO-OFDM CP design is identical to the ACO-OFDM system as will be illustrated in the following section.

Figure 1 shows a block diagram for applying spatial DC bias of the OFDM system and is used to rise the IDFT output to a minimum amplitude greater than zero. In comparison to ACO-OFDM, this technique of adding DC-bias to DCO-OFDM is used to apply all negative values to zero. The clipping and ranging suggest maintaining the peak-to-average power ratio (PAPR) fluctuations and scale the clipped values ready to be transmitted [18].

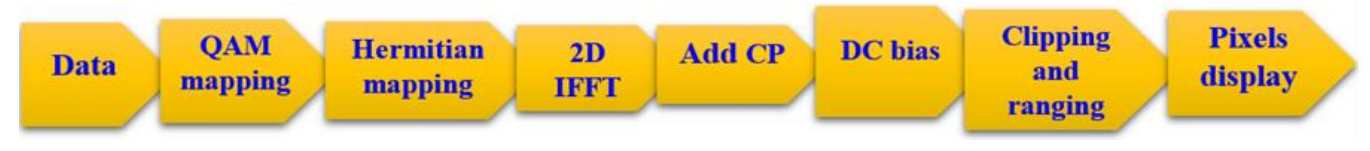

Figure 1. Transmitter part of DCO-OFDM system [18]

Assuming that the total number of $\mathrm{N}$ subcarriers is assigned to a single block of OFDM, in which $\mathrm{N}$ is typically an even large number. For special constellation modulation $\mathrm{X}$, such as quadrature amplitude modulation (QAM). The serial bit stream is first transformed to the parallel sequence at the transmitter and mapped to the complex-valued symbols N/2-1. The modulated OFDM symbol is assembled as follows $\mathrm{X}=[\mathrm{X} 0 \mathrm{X} 1 \cdots \cdots \mathrm{XN}-1]$, where the subscript number $(*)$ denotes the subcarrier conjugate associated with it:

$\mathrm{X} 0=0$ and $\mathrm{X} 1$ to $\mathrm{XN} / 2-1$ are symbolized with $\mathrm{N} / 2-1$ details, while $\mathrm{XN} / 2$. In $\mathrm{XN}-1$ the Hermitian symmetry satisfies as (1):

$$
X_{K}=X^{*}{ }_{N-K}, k=\frac{N}{2}, \ldots \ldots N-1,
$$

Choice $\mathrm{X} 0=\mathrm{XN} / 2=0$ is designed to prevent $\mathrm{DC}$ and complex harmonic components. The Hermitian $\mathrm{X}$ symmetry allows the transmitter to produce real-time-value signals [18]. The OFDM symbol vector $\mathrm{X}$ is provided for the inverse fast Fourier transform (IFFT) and effectively converted to discrete time-domain samples as (2),

$$
x_{n}=\frac{1}{\sqrt{N}} \sum_{k=0}^{N-1} X_{k} \exp \left(j \frac{2 \pi k n}{N}\right), n=0,1, \ldots . N-1,
$$

where $x_{n}$ shows the $n t h$ discrete time-domain samples.it is considered the imposed Hermitian symmetry, the operation of the IFFT can be expressed as (3),

$$
x_{n}=\frac{1}{\sqrt{N}} \sum_{k=1}^{N / 2-1}\left(X_{k} \exp \left(j \frac{2 \pi k n}{N}\right)+X_{N-K} \exp \left(j \frac{2 \pi k(N-K) n}{N}\right)\right)
$$

where $\operatorname{Re}(Z)$ corresponds to the actual $Z$ part. The imaginary sections of the signal samples of the time-domain are applied to zero. It should be noticed that the overall amount of subcarriers used is N/2-1 and the remaining subcarriers would be used to implement the Hermitian symmetry [18].

\subsection{D ACO-OFDM}

The functional block of ACO OFDM is defined as follows, particularly for the screen ACO-OFDM system as shown in Figure 2, a practical block diagram for implementing spatial ACO-OFDM is designed to map the sequence of bits to a complex sequence of numbers (one-dimensional sequence).Hermitian mapping 
structural design of a bi-dimensional matrix of complex numbers from a sequence of complex numbers in such a way as to ensure that the 2D-IDFT is entirely real [18].

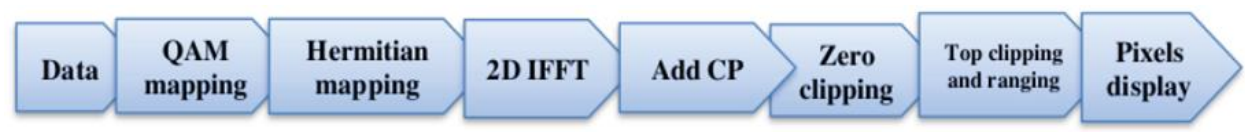

Figure 2. Transmitter part of DCO-OFDM system [18]

Data symbols are only mounted on the odd subcarriers of the first N/2 subcarriers in the ACO-OFDM scheme and can thus only support N/4 information symbols in the ACO-OFDM block of N subcarriers. The Hermitian symmetry limitation of (5) is also imposed on the $(\mathrm{N} / 2)$ th to $(\mathrm{N}-1)$ th subcarriers in terms of the limitation of real-value amplitude. After the IFFT process, at the transmitter, the discrete time-domain samples have the asymmetry as [19].

$$
\begin{aligned}
& x_{n}=\frac{1}{\sqrt{N}} \sum_{k=0}^{N-1}\left(X_{k} \exp \left(j \frac{2 \pi k n}{N}\right)\right) \\
& =x_{n}+\frac{N}{2}, n=0,1, \ldots N-1
\end{aligned}
$$

The equation derivation of (5) was introduced in [19]. The asymmetry in (7) displays the information that the amplitudes of the first half samples are matching to the second half samples but with opposite signs. The negative-valued sample $x_{n}<0$ can be just improved by detecting its asymmetry sample $x_{n}+N / 2$ or $x_{n}-N / 2$.Hence, the ACO-OFDM signal $x$ ACO $(t)$ is directly clipped at zero without adding any DC bias and then changed into an optical signal. Supposing that $x_{n}^{c}$ is the clipped sample, where (6):

$$
x_{n}^{c}=\left\{\begin{array}{c}
x_{n} \text { if } x_{n}>0 \\
0 \text { if } x_{n}<0
\end{array}\right\}
$$

The distorted data symbols $x_{2 m+1}^{c}$ on the odd subcarriers $m=0,1 \ldots N / 2-1$ can be expressed as:

$$
\begin{aligned}
x_{2 m+1}^{c} & =\frac{1}{\sqrt{N}} \sum_{n=0}^{N-1}\left(x_{n}^{c} \exp \left(-j \frac{2 \pi(2 m+1) n}{N}\right)\right) \\
& =\frac{1}{2} x_{2 m+1}
\end{aligned}
$$

It can be shown that the ACO-OFDM power signal is reduced significantly by the clipping technique [19].

\section{SYSTEM SETUP}

The simple block diagram for the 2D OFDM OCC system is shown in Figure 3 which illustrates the performance analysis of the optical OFDM system. A bidirectional optical OFDM OCC framework has been implemented in this research. Figure 4 presents the transmitter code for the encoded data which indicates that the display size of the configurable transmitter as each of the tiny cells.

Figure 3 indicates that the design of the transmitter comprises 2 OFDM optical modes that have been added which are ACO-OFDM and DCO-OFDM for the investigation of the BER performance relationship between them. The reliability of the PAPR reduction schemes can be calculated based on two factors that increase the clipping factor for the optical OFDM systems, the coded and encoded performance of the BER [20]. Besides, the functional clock rate for adjusting the transmitter processing speed [21]. Detailed parameters used for the OCC system are presented in Table 1 which gives information about OFDM symbol size and the max number of the MIMO Frames. This table also shows the Hermitian mapping used at three different clipping factors $0.7,1.4$ and 2.6 with SNR starts at $8 \mathrm{~dB}$. Figure 5 displays the receiver decoded text with and without noise. It is known that $\mathrm{CP}$ aims to minimize interference between OFDM symbols [22]. 


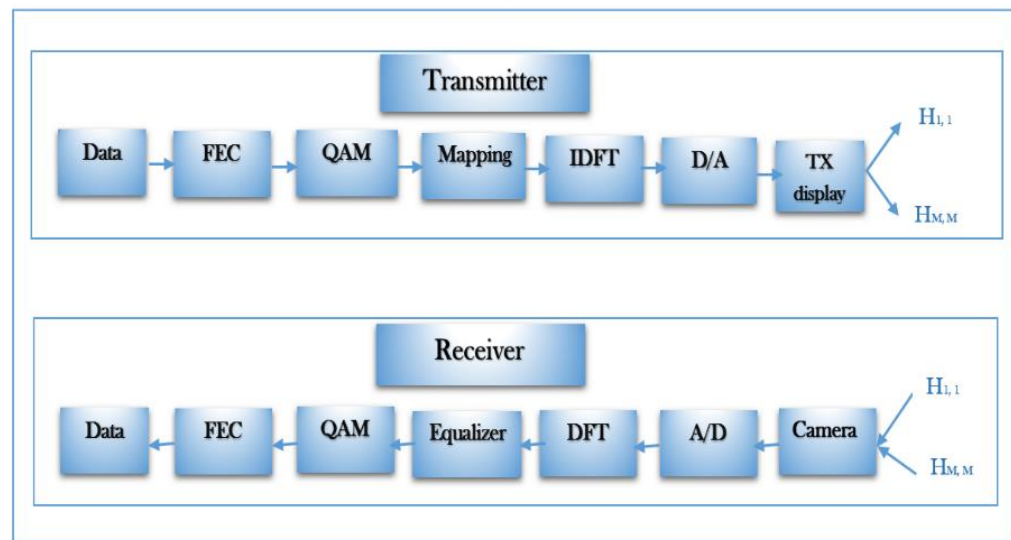

Figure 3. Proposed optical OFDM OCC system

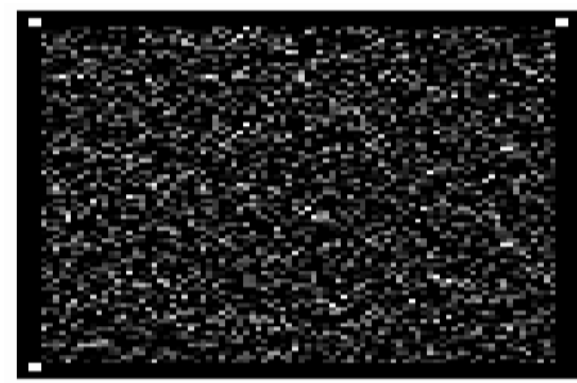

Figure 4. Tx code for OCC system

Table 1. OCC system parameters

\begin{tabular}{cccccccc}
\hline $\begin{array}{c}\text { OFDM } \\
\text { symbol size }\end{array}$ & $\begin{array}{c}\text { Number of } \\
\text { Tx cells }\end{array}$ & $\begin{array}{c}\text { Hermitian } \\
\text { mapping }\end{array}$ & $\begin{array}{c}\text { Max number of } \\
\text { MIMO frames }\end{array}$ & $\begin{array}{c}\text { no. of bit per } \\
\text { sub transmitter }\end{array}$ & $\begin{array}{c}\text { clipping } \\
\text { factors }\end{array}$ & SNR & $\begin{array}{c}\text { the inner } \\
\text { part of Tx }\end{array}$ \\
\hline $28 \times 28$ & $96 \times 96$ & $\begin{array}{c}\text { ACO\&DCO } \\
\text { OFDM }\end{array}$ & 27 & 236 & $0.7,1.4,2.6$ & Start at $8 \mathrm{~dB}$ & $84 \times 84$ \\
\hline
\end{tabular}

(Noisy link test) RX decoded Text

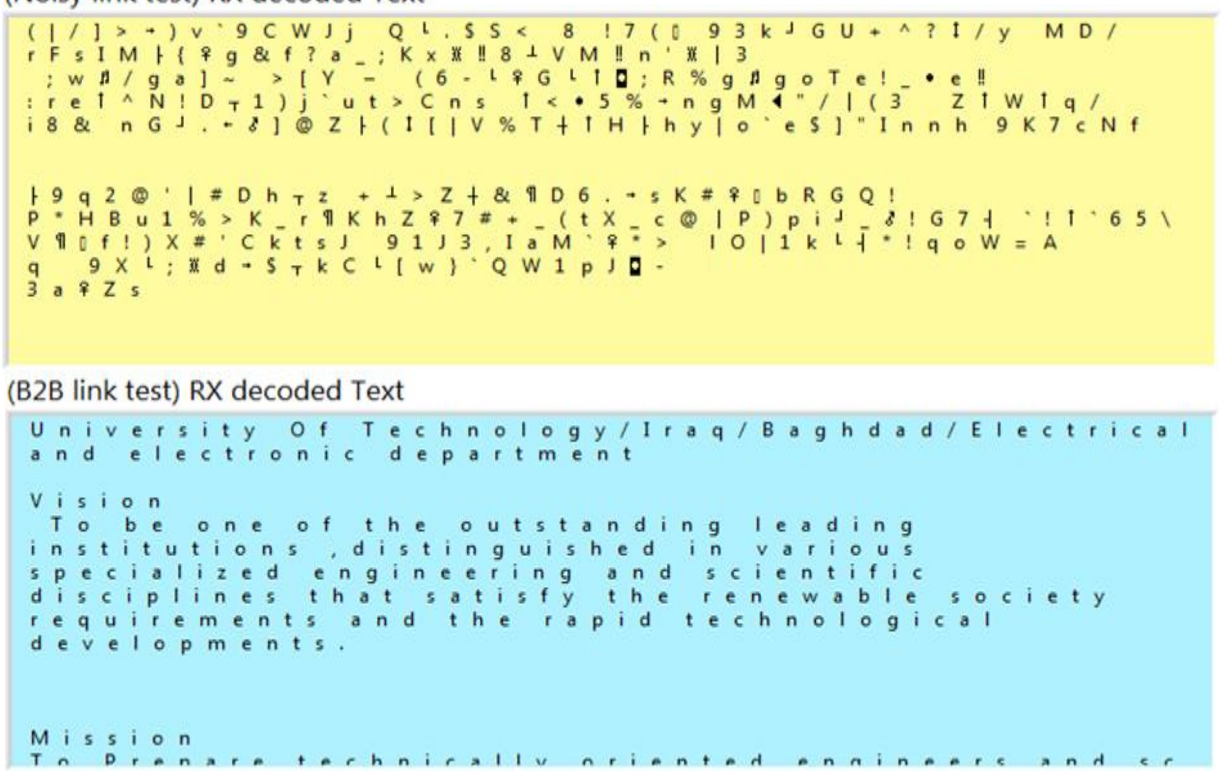

Figure 5. The receiver decoded text with and without noise 
In general, the reliability of PAPR reduction techniques can be assessed in terms of the various two perspectives which change the clipping factor for ACO-OFDM and DCO-OFDM framework [23], BER reliability coded and encoded. The relationship between ACO-OFDM and DCO-OFDM using the BER versus the SNR for data transfer is also discussed in this section. The communication system bit error rate is described as the measure of the number of bit errors to the total number of bits sent over a specified period [24]. Among received bits, a single bit of error might well happen irrespective of the rate of transmission. SNR is known as the signal-to-noise ratio and is commonly defined in decibel $(\mathrm{dB})$.

\section{SIMULATION RESULTS AND ANALYSIS}

Two kinds of optical OFDM and BER versus SNR for data transfer will be addressed in this section. The clipping factors can be introduced by 8-bit value pixels to control PAPR. The BER efficiency of the DCO OFDM and ACO OFDM at three separate clipping factors $0.7,1.4$, and 2.6 is presented in Figure 6(a) and Figure 6(b). The choice of clipping factors is essential and has a significant impact on the implementation results. Based on the SNR, the clipping factor was adjusted to an acceptable BER. The implementations of two layers, layer one without FEC and layer two with convolutional code CC FEC.
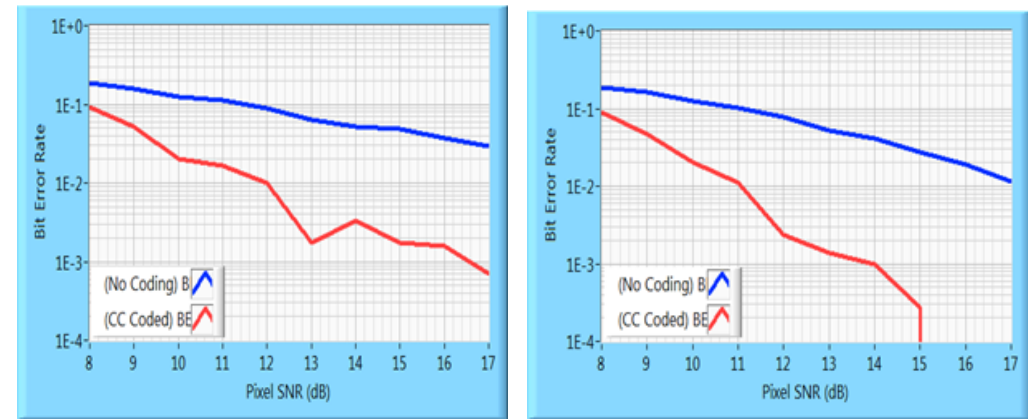

(a)
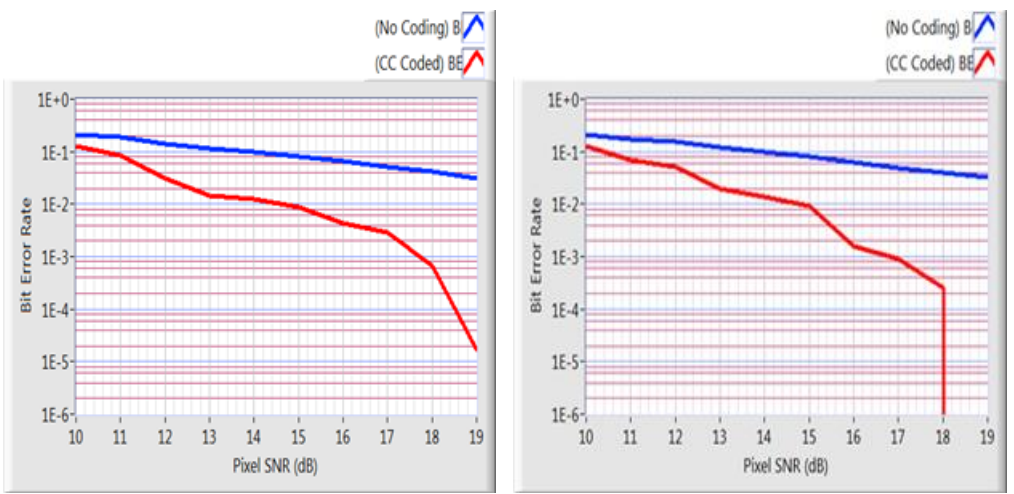

(b)
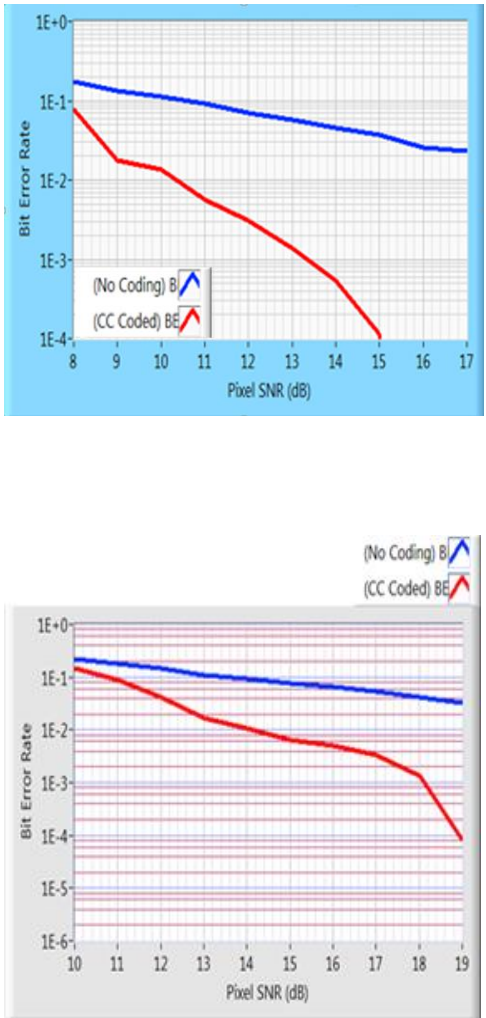

Figure 6. BER performance comparison of; (a) ACO OFDM, and (b) DCO OFDM at three clipping factors $0.7,1.4$, and 2.6 respectively

It is really important to select the clipping factor which has a direct impact on system performance. It is found that there would be a decrease in PAPR output after rising the clipping factor as it can be shown that the BER at 1.4 clipping factor was $10^{-4}$ while BER will be $10^{-6}$ at 2.6 clipping factors at the expense of SNR around $18 \mathrm{~dB}$ signal to noise ratio. This is that for a certain number of samples there was no error threshold, there is an SNR threshold for making the error [25].

After increasing the number of higher precision loops, it is observed that the accuracy as seen in Figure 6 has been increased. The clipping process is the simplest technique for reducing the PAPR, which decreases the overall amount of signal transmission to a pre-specified level. However, clipping introduces inband signal distortion with the following drawbacks, resulting in BER output degradation [26]. 
It is noticeable from Figure 6 is measurable that after increasing the clipping factor to 1.4, SNR would be $15 \mathrm{~dB}$ and BER is $10^{-6}$, which is higher than the BER output compared to [18], the BER efficiency of the ACO OFDM will increase. It is often shown that a drop in BER can be caused by too low a clipping factor or too high. The choice of clipping levels from the result is very critical and has a direct impact on the efficiency of the system.It is seen from the results of the simulation that ACO OFDM efficiency is more stable than the DCO-OFDM model when comparing the functional BER curves. Figure 6 describes the measurements of BER of optical-OFDM under the impacts of clipping variables. It is observed:

- PAPR decreased at the expense of increasing the BER bit error rate, increasing computational performance such as coding and encoding for the ACO OFDM system.

- Compared to the ACO OFDM, the DCO OFDM has the best clipping factor at 1.4 will acquire approximately $2 \mathrm{~dB}$ SNR gain and BER is $10^{-6}$.

- The clipping factor choice is significant and has a direct effect on system performance.

- All results indicate that the lower the clipping ratio, the better the performance of the PAPR reduction, but the worse the bit error rate efficiency.

- The ACO-OFDM gain of $3 \mathrm{~dB}$ is because it uses just half as many two-dimensional subcarriers as DCOOFDM for data transfer.

\section{CONCLUSION}

Two kinds of optical OFDM systems have been introduced in this study. The BER performance of the ACO OFDM and DCO OFDM systems has also been presented. It is observable that the best selection to the clipping ratio leads to minimizing the SNR to measure the effect on the BER system performance. Simulated results include the process of choosing with the optimum clipping ratio that satisfies SNR gain consumed in ACO OFDM and DCO OFDM systems.

In the situations of low SNR, it is assumed that a lower clipping factor is better. The DCO OFDM output is more robust than the ACO-OFDM system when the BER curves are compared. From the final results, it is important to select the clipping factors and have a straight affect on the system performance. If the clipping ratio decreases when the signal experiences more and more nonlinear distortion, the output of the BER versus the obtained SNR gets worse and worse.

The major challenge discussed in this paper is to check that the signal was produced correctly and what variables would influence the output of the system if the back-to-back transmitter and receiver were linked. It is also suggested that the greater the clipping noise will decrease the PAPR by reducing the clipping ratio to $10^{-6}$ but will benefit SNR at the BER decrease (dB). BER accuracy for both modes of optical OFDM can be increased with CC FEC.

\section{ACKNOWLEDGEMENTS}

I would like to thank my supervisor who has helped me at any point in my research work. I would also like to express my gratitude and thanks to the optical press team of those who supported me at every point of my research work, my friends, for their unending love and care. And God, the almighty, for helping me fight all the obstacles that have come to me.

\section{REFERENCES}

[1] C. W. Chow et al., "Enabling Techniques for Optical Wireless Communication Systems," Optical Fiber Communication Conference Optical Society of America, 2020, pp. 1-3, doi: 10.1364/OFC.2020.M2F.1.

[2] T. Nguyen, A. Islam, T. Hossan, and Y. M. Jang, "Current Status and Performance Analysis of Optical Camera Communication Technologies for 5G Networks," IEEE Access, vol. 5, pp. 4574-4594, 2017, doi: 10.1109/ACCESS.2017.2681110.

[3] T. Nguyen, A. Islam, T. Yamazato, and Y. M. Jang, "Technical Issues on IEEE 802.15.7m Image Sensor Communication Standardization," IEEE Communications Magazine, vol. 56, no. 2, pp. 213-218, Feb. 2018, doi: 10.1109/MCOM.2018.1700134.

[4] Y. Li, D. Tsonev, and H. Haas, "Performance Analysis of Non-DC-Biased OFDM," arXiv: 1905.05822, May 2019.

[5] T. Zhang, J. Yao, and S. Guo, "The Novel PAPR Reduction Schemes for O-OFDM-Based Visible Light Communications,” in Visible Light Communications, pp. 89-110, Jul. 2017.

[6] S. D. Dissanayake and J. Armstrong, "Comparison of ACO-OFDM, DCO-OFDM and ADO-OFDM in IM/DD Systems,” J. Lightwave Technol., vol. 31, no. 7, pp. 1063-1072, Apr. 2013.

[7] N. J. Jihad and S. M. A. Satar, "Optical Camera Communication Performance Evaluation: Review," Iraqi Journal of computers, communication, control and systems engineering, vol. 20, no. 3, pp. 42-49, 2020, doi: 10.33103/uot.ijccee.20.3.4. 
[8] N. J. Jihad and S. M. A. Satar, "OCC Future and Obstacles under 5G Requirements," Emirates Journal for Engineering Research, vol. 25, no. 3, 2020, Art. No. 6.

[9] A. W. Azim, Y. Le Guennec, and G. Maury, "OFDM for optical wireless systems under severe clipping conditions," in 2016 Advances in Wireless and Optical Communications (RTUWO), Riga, Latvia, Nov. 2016, pp. 201-206, doi: 10.1109/RTUWO.2016.7821884

[10] R. Alindra, M. O. Fauzan, R. Ramadhan, and S. Rahardjo, "Performance Analysis of DCO-OFDM and ACO-OFDM for Visible Light Communication System," in 2018 3rd International Seminar on Sensors, Instrumentation, Measurement and Metrology (ISSIMM), Depok, Indonesia, Dec. 2018, pp. 84-90, doi: 10.1109/ISSIMM.2018.8727737.

[11] Md. Shahjalal, Moh. K. Hasan, M. Z. Chowdhury, and Y. M. Jang, "Smartphone Camera-Based Optical Wireless Communication System: Requirements and Implementation Challenges," Electronics, vol. 8, no. 8, pp. 1-17, Aug. 2019, Art. No. 913, doi: 10.3390/electronics8080913.

[12] T. Nguyen, N. T. Le, and Y. M. Jang, "Asynchronous Scheme for Optical Camera Communication-Based Infrastructure-to-Vehicle Communication," International Journal of Distributed Sensor Networks, vol. 11, no. 5, pp. 1-11, May 2015, doi: 10.1155\%2F2015\%2F908139.

[13] S. Dixit and D. Nagaria, "LMS Adaptive Filters for Noise Cancellation: A Review," International Journal of Electrical and Computer Engineering (IJECE), vol. 7, no. 5, pp. 2520-2529, 2017, doi: 10.11591/ijece.v7i5.pp25202529.

[14] J. K. Hmood, K. A. Noordin, S. W. Harun, and H. M. H. Shalaby, "Mitigation of phase noise in all-optical OFDM systems based on minimizing interaction time between subcarriers," Optics Communications, vol. 355, pp. 313-320, Nov. 2015, doi: 10.1016/j.optcom.2015.06.059.

[15] J. K. Lain, Z. D. Yang, and T. W. Xu, "Experimental DCO-OFDM Optical Camera Communication Systems with a Commercial Smartphone Camera," IEEE Photonics Journal., vol. 11, no. 6, pp. 1-13, Dec. 2019, doi: 10.1109/JPHOT.2019.2948071.

[16] S. Dimitrov, S. Sinanovic, and H. Haas, "Clipping Noise in OFDM-Based Optical Wireless Communication Systems," IEEE Transactions on Communications, vol. 60, no. 4, pp. 1072-1081, Apr. 2012, doi: 10.1109/TCOMM.2012.022712.100493.

[17] L. A. AL-Hashemi, G. A. Al-Suhail, S. M. Abdul Satar, A. N. Kareem, and M. A. Hussein, "An ICI Reduction Based on PAPR Clipping in Coherent Optical OFDM System," in 2018 International Conference on Advanced Science and Engineering (ICOASE), Duhok, Oct. 2018, pp. 244-249, doi: 10.1109/ICOASE.2018.8548855.

[18] T. Nguyen, M. D. Thieu, and Y. M. Jang, "2D-OFDM for Optical Camera Communication: Principle and Implementation," IEEE Access, vol. 7, pp. 29405-29424, 2019, doi: 10.1109/ACCESS.2019.2899739.

[19] Z. Wang, Q. Wang, W. Huang, and Z. Xu, "Visible light communications: modulation and signal processing," Hoboken, New Jersey, John Wiley and Sons, Inc, 2017.

[20] N. J. Jihad and S. M. A. Satar, "Performance study of ACO-OFDM and DCO OFDM in optical camera communication system," in 2020 2nd Al-Noor International Conference for Science and Technology (NICST), 2020, pp. 63-67, doi: 10.1109/NICST50904.2020.9280319.

[21] M. H. Ali, A. H. Ali, S. M. Abdulsatar, M. A. Saleh, A. K. Abass, and T. F. Al-Mashhadani, "Pump power optimization for hybrid fiber amplifier utilizing second-order stimulated Raman scattering," Opt Quant Electron, vol. 52, no. 6, Jun. 2020, Art. No. 274, doi: 10.1007/s11082-020-02400-x.

[22] L. A. Al-Hashime, G. A. Al-Suhail, and S. M. A. Satar, "Modulation Mapping Influence in Coherent Optical OFDM System for Long Haul Transmission," in International Conference on New Trends in Information and Communications Technology Applications, 2018, pp. 193-209, doi: 10.1007/978-3-030-01653-1_12.

[23] N. B. Hassan et al., "Impact of Camera Lens Aperture and the Light Source Size on Optical Camera Communications," in 11th International Symposium on Communication Systems, Networks and Digital Signal Processing (CSNDSP), Budapest, Jul. 2018, doi: 10.1109/CSNDSP.2018.8471766.

[24] P. Varzakas, "Optimization of an OFDM Rayleigh fading system," International Journal of Communication Systems, vol. 20, no. 1, pp. 1-7, 2007, doi: 10.1002/dac.807.

[25] N. Saeed, S. Guo, K. H. Park, T. Y. Al-Naffouri, and M. S. Alouini, "Optical camera communications: Survey, use cases, challenges, and future trends," Physical Communication, vol. 37, pp. 1-17, Dec. 2019,

[26] M. H. Ali, A. K. Abass, and S. A. Abd Al-Hussein, "32 Channel $\times 40 \mathrm{~Gb} / \mathrm{s}$ WDM optical communication system utilizing different configurations of hybrid fiber amplifier," Opt Quant Electron, vol. 51, no. 6, Jun. 2019.

\section{BIOGRAPHIES OF AUTHORS}

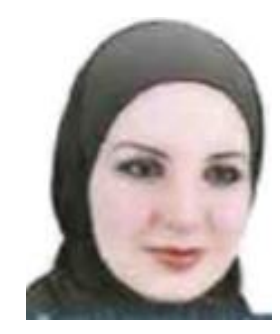

Noor J. Jihad was born in Baghdad, Iraq in 1987. She received her B.Sc. degree from the University of Technology (First Rank) in electrical and electronics engineering in 2009. She received an M.Sc. degree with distinction in communications engineering from Brunel University London in 2012. She has been a Lecturer at the University of Technology, Communication Engineering Department from 2014 until now. Currently, she joined a Ph.D. study at the Faculty of Electrical and Electronics Engineering, University of Technology, Iraq. Her research interests: optical camera communication systems, optical wireless communication systems, optical fiber communications engineering systems, modern communications systems, wireless and microwave engineering. Noor is an IEEE member since 2019. 


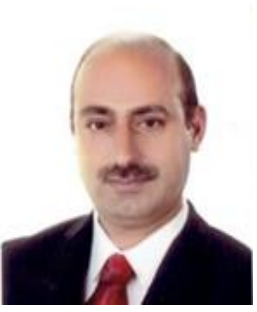

Sinan M. Elias was born in Baghdad, Iraq in 1970. He received his B.Sc. and M.Sc. degrees in 1993 and 1998 respectively from MEC, Iraq. From 2003-2006, he joined a Ph.D. study at the Faculty of Electrical and Electronics Engineering, University of Technology, Iraq. Since 2012, he has been an Assistant Professor of Electronics and Communications Eng. At the UOT, Iraq. From 2018 until now, Dr. Elias headed the Electronic branch at the electrical department, University of technology. He has been a Professor of Electronics and Communications Eng. at the UOT, Iraq in 2020. He started scientific publishing in 2000, he has more than 37 publications in national and international conferences and journals. Dr. Sinan is IEA and ILS member. 